\title{
Using WOFOST crop model for data base derivation of tritium and terrestrial food chain modules in RODOS
}

\author{
A. Melintescu, D. Galeriu and A. Marica ${ }^{1}$ \\ National Institute for Physics and Nuclear Engineering, \\ P.O. Box MG-6, 76900 Bucharest-Magurele, Romania \\ ${ }^{1}$ National Institute for Meteorology and Hydrology, Bucharest, Romania
}

\begin{abstract}
The European Commission Project RODOS is developing a coherent methodology for a Real-time On-line DecisiOn Support System for Nuclear Emergency across Europe. Within this system there is a special module to model the transfer of triated water from releases to terrestrial foods. In order to model the transfer of tritiated water from air to various plants, the conversion to organically bound tritium, and the partition to edible parts of the plant, both the mean dynamics of leaf area index and a physiological description of canopy photosynthesis are required. The WOFOST crop growth model has been selected as a basis for deriving tritium transfer dynamics to plants. Its ability to reproduce site-specific biomass growth of various plants (not only from Europe) is demonstrated in this paper, as well as its compatibility to other photosynthesis models. We have tested that this model can simulate limited fertilisation situations via the adaptation of two important parameters. After adaptation of model parameters to sitespecific plant growth data, multi-annual mean dynamics can be obtained using meteorological data for subsequent years.
\end{abstract}

\section{INTRODUCTION}

Under the auspices of its Research and Technological Development Framework Programmes, the European Commission has supported the development of the RODOS (Real-time On-line DecisiOn Support) system for off-site emergency management. In 1997 it was realised that tritium should be included in the list of radionuclides, as large tritium sources exists in Europe and to allow a potential expansion of the RODOS system for application to future fusion reactor accidents. Tritium, is incorporated immediately in the hydrogen cycle and consequently its transport into the biosphere differs considerably from other radionuclides treated by the RODOS system. Therefore, the tritium code in RODOS was developed as a separate, although compatible, module [1]. A common requirement of both the tritium and food chain modules in RODOS is the need to know the mean dynamics of leaf area index (LAI)] of various plants, as well as the final crop yield. For modelling tritiated water transfer from air-soil to plant, and the conversion to organically bound tritium (OBT), we need to assess canopy photosynthesis. The formation rate of OBT during daylight is proportional to the photosynthesis rate. The flux of HTO from air to leaf is dependant upon canopy resistance. Canopy resistance is estimated as the ratio between canopy photosynthesis rate and the $\mathrm{CO}_{2}$ concentration gradient from air to internal leaf [23]. The tritium model must be applicable to the various ecological regions of Europe [and preferably world-wide], with a minimum of local calibration. We expect a greater predictive ability if tritium models incorporate scientific knowledge and readily accessible experimental data on plant physiology. . Consequently, the photosynthesis sub model, required to drive tritium transfer, must be physiologically robust, easy to customise and respond to fast changes in light and temperature.

\section{WOFOST crop growth model as a driving model for tritium transfer}

After detailed analyses of the many crop growth models available with respect to their ability to respond to diurnal forcing and the comprehensiveness of their databases, it was decided to use the canopy photosynthesis model from the WOFOST crop growth code [4]. This has a large database and can be operated at an hourly time step. Within WOFOST leaf gross photosynthesis rate $\left(\mathrm{A}_{\mathrm{Lg}}\right)$ is given by:

$$
A_{\mathrm{Lg}}=A_{\mathrm{gm}} *\left(1-\exp \left(-\varepsilon^{*} I_{\mathrm{aI}} / A_{\mathrm{gm}}\right)\right)
$$

Where the maximum gross photosynthetic rate $\left(A_{\mathrm{gm}}\right)$ depends on crop type, age and ambient temperature; the initial light use efficiency $(\varepsilon)$ [slope of photosynthesis rate at low light intensity] depends on crop type 
but the weak dependency of this parameter on temperature is ignored within WOFOST. $A_{\text {gm }}$ also depends on internal leaf concentration of $\mathrm{CO}_{2}$ (determined by ambient concentration, boundary and stomata conductance). The absorbed photosynthetically active radiation (PAR), $\mathrm{I}_{\mathrm{aL}}$ is separately assessed for leaves in sunlight and shade and for direct and diffuse incident light. The associated extinction coefficient of light in the canopy depends on plant type. WOFOST contains a large database for various crops and cultivars, from which the above parameters were selected to reproduce crop growth under conditions of unlimited fertilization.

In order to assess the uncertainty of our model predictions for ${ }^{3} \mathrm{H}$ we need to analyse: 1) how WOFOST model compares with more complex biochemical photosynthesis models; 2) how model parameters can be adjusted for conditions where fertilisation is rate limiting; 3) how model parameters can be established for cultivars and plants not included in the WOFOST data base.

\subsection{Reliability of WOFOST photosynthesis model}

An analysis of factors influencing the reliability of photosynthetic rate assessment [5] shows that consideration of direct and diffuse light and distinction between leaves in shade and sunlight are essential, Because $A_{\mathrm{gm}}$ depends on the amount of light and leaf age, the canopy photosynthesis must consider the canopy depth dependence of $\mathrm{A}_{\mathrm{gm}}$ (i.e. multilayered canopy). When using a bulk value (canopy average), this must be accompanied by a bulk value for the boundary layer conductance in order to give the right value in comparison to a multilayered model [5]. Analysing the WOFOST photosynthesis submodel in respect with above requirements we conclude that this are included so the bulk canopy approach in WOFOST, together with a bulk boundary layer resistance value (when assessing canopy resistance), gives comparable prediction to a multilayer approach., if the leaf photosynthesis model is appropriate.

Equation [1] is only one possible photosynthesis model. For wheat a nonrectangular hyperbola model based on experimental data has previously been used [6] and this model and parameters was used in the WOFOST routine, in parallel with the exponential model. The same irradiance (diffuse, direct) and leaf partition (shaded, sun-light) values were used to compare the predictions of the default WOFOST exponential model with the non-rectangular hyperbola. Figure 1 demonstrates that although the WOFOST predictions are consistently higher that those of the non-rectangular hyperbola model the overestimate was $<10 \%$.

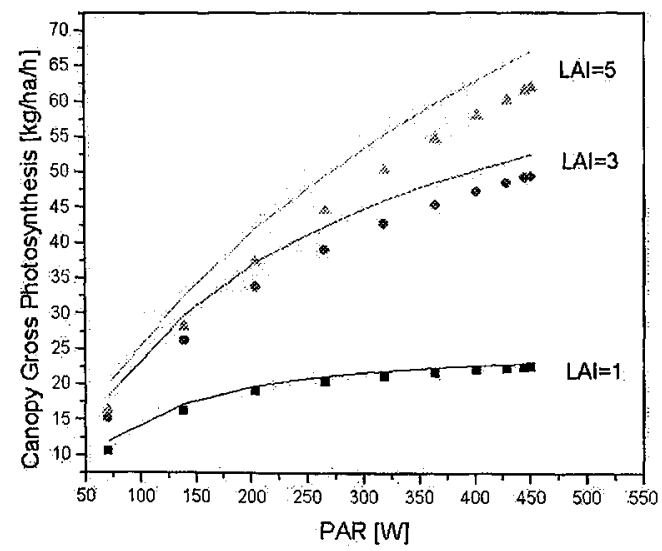

Figure 1 (left) Comparison between nonrectangular hyperbola [symbols] and exponential WOFOST model [lines] for wheat canopy photosynthesis at LAI values of 1,3 and 5 .

The WOFOST database contains only tentative data for grass. For model application we need to be able to simulate intensively and extensively managed grasslands of C3 and C4 types. For a composite of $\mathrm{C} 4$ wild grass species, an advanced biochemical model carefully fitted to experimental data has been published [7]. The predictions of this model were successfully fitted to Equation (1). The resulting 
WOFOST parameters for natural C4 grass species in environments typical of Southern USA reflects the extensive, nonfertilized situation: the maximum rate of $\mathrm{A}_{\mathrm{gm}}$, is $45 \mathrm{~kg} \mathrm{CO} / \mathrm{ha} / \mathrm{h}$, (c.f the unlimited fertilization value of 70 for generic C4 plants used in WOFOST) and the fitted light use efficiency of 0.3 is less than optimal production one.

Maize is a C4 plant originating in America, although adapted to European conditions for a long time. The WOFOST database contains many maize cultivars for Europe but we are also interested in testing the applicability to other continents. A bio-kinetic model for $\mathrm{C} 4$ plants has previously been reported [8] with parameters adapted to maize cultivars from Southern USA. We have reconstructed the model and produced a set of data on leaf photosynthesis at various temperatures. The generated data were fitted to Equation (1) and good agreement was obtained with the following parameters: $A_{\mathrm{gm}}=74 \mathrm{~kg}$ $\mathrm{CO}_{2} / \mathrm{ha} / \mathrm{h}$ and $\varepsilon=0.32$ (compared with the European cultivars having $\mathrm{Agm}_{\mathrm{gm}}=74 \mathrm{~kg} \mathrm{CO} / \mathrm{ha} / \mathrm{h}$ and $\varepsilon=0.45$ ). Relative to European maize, the US cultivar has the same $A_{g m}$ but the temperature dependence differs mainly between 10 and $30^{\circ} \mathrm{C}$. We conclude that the WOFOST model can reproduce plant growth in nonEuropean environment, with parameter adaptation. Relative to advanced bio-chemical models of photosynthesis (Farquarhad type), we have found that the WOFOST approach give comparable results after parameter adaptation, as tested by us on grass, maize and other cases.

\subsection{Fertilisation limited crop production}

The WOFOST model was designed to predict potential crop production rates. It has the option of limited water supply, unlimited (i.e. optimal agricultural management) fertilization or natural ecosystem soils with low fertilization. In developed countries the agricultural practice is general close to optimal but for other area, fertilization is far for optimum. Many crop growth models include variable fertilization of soil, at least nitrogen. Added fertiliser is dissolved in the soil solution and also fixed in inert forms (mineralisation) depending on soil properties. Because we are not interested in detailed process, we consider only the degree of fertilisation influencing the nitrogen uptake by plant. It is well established [9], that the photosynthesis rate depends directly on the leaf nitrogen content with a saturating response. Leaf nitrogen content also affects the leaf thickness, or in terms of WOFOST, the specific leaf area (SLA). A decreased nitrogen supply will decrease $A_{g m}$ and will increase SLA.
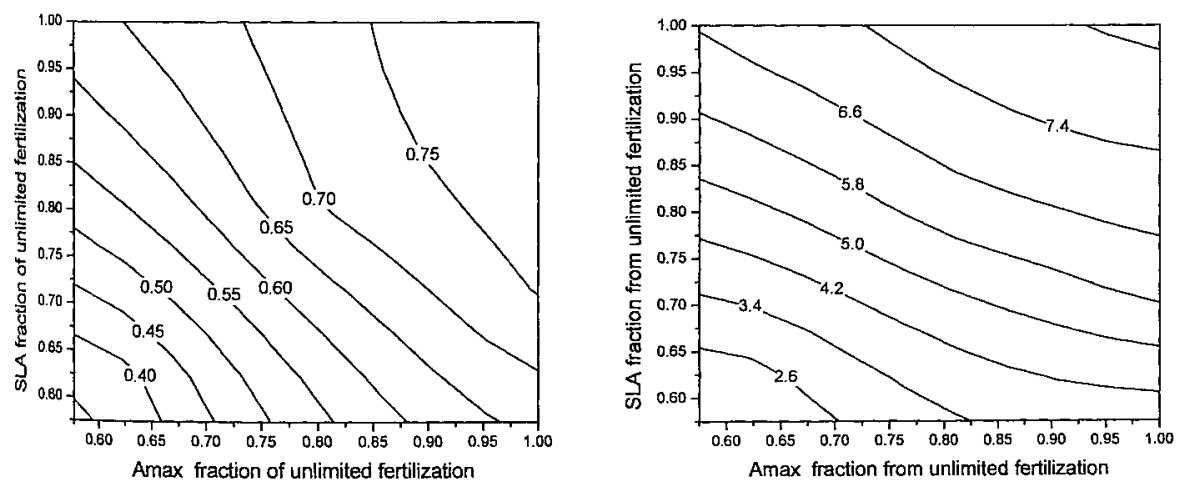

Figure 2 Wheat yield dependence (left) and maximum $\mathrm{LAI}$ (right) on the ratios $\mathrm{A}_{\mathrm{MAX}}=\mathrm{A}_{\mathrm{gm}}$ (actual)/ $\mathrm{A}_{\mathrm{gm}}$ (potential) and SLA $=$ SLA(actual)/SLA(potential), for simulating limited fertilisation

If we define the maximum yield and LAI under unlimited fertilization, as given by the default parameters in WOFOST, we can reproduce crop production under conditions of lower fertilization by altering $A_{\mathrm{gm}}$ and SLA in a coherent manner: knowing the maximum yield and leaf area under unlimited fertilization, and the measured yield and maximum LAI, we can optimise the model parameters using the known dependence of these quantities on $\mathrm{Agm}_{\mathrm{gm}}$ and SLA.

An example is given in Figure 2 for wheat where two-dimensional plots of yield (left) and maximum LAI (right) are depicted as the ratio of actual $A_{g m}$ and SLA with the nominal ones, for unlimited fertilisation. 
With at least 4-6 experimental data points for LAI and biomass dynamics in a specific year and known daily meteorology, the relationships within Figure 3 enable the WOFOST parameters to be customised for limited fertilization.

\subsection{Application: Establishing model (WOFOST) parameters for South Romania}

Maize, wheat, sunflower, potato and grapes are the major crops close to the Southern Romanian CANDU reactor, which represents a tritium source. With the exception of grapes these plants are included in the WOFOST database, although for cultivars grown in other areas of Europe. There is no a priori reason to believe that the cultivars in WOFOST database are appropriate for South Romania. Agricultural research institutes have supplied experimental data from South Romania on biomass and LAI dynamics for specific years, together with soil and climate data.

Sunflower is a interesting plant in terms of ${ }^{3} \mathrm{H}$ as the product, oil, contains no water and consequently OBT concentrations could be high. Data on biomass and LAI dynamics, seedling, emergence, anthesys, maturity and 12 time dependent values of above ground and storage organ mass, as well LAI for 1986 were obtained for the two main cultivars in South Romania. From the database in WOFOST we selected, as a starting point, a South European cultivar. We ran WOFOST with the data supplied for daily weather and modified the following parameters so that predictions better fitted the observed data: temperature sum [degree-days] from seedling to emergence, emergence to anthesis and anthesis at maturity; specific leaf area; dynamics of partitioning to storage organs. Generally the parameters were modified by less than $20 \%$ from the original values. An example of model results compared with experimental data for above ground biomass is given in Figure 3. Similar experimental data were obtained for three cultivars of maize, in 1989. We used as a starting data WOFOST cultivars from North Italy and we modified the temperature sum for phenologic phase and the dynamics of partition to storage organs. An example for LAI dynamics is given in Figure 4 for the experimental data (exp LAI), the default WOFOST predictions (WCC LAI) and predictions obtained with a composite model developed by us (DG LAI). Similar procedures were conducted for potato and winter wheat. For winter wheat, the effect of limited fertilization, typical of Romania, is greater in non-irrigated situations (a reduction at $50 \%$ in yield) compared with irrigated situations (only a $20 \%$ reduction in yield from $400 \mathrm{~N}$ to $0 \mathrm{~N}$ ). These effects can be easily reproduced in WOFOST by altering $\mathrm{A}_{\mathrm{gm}}$ and SLA as described above.
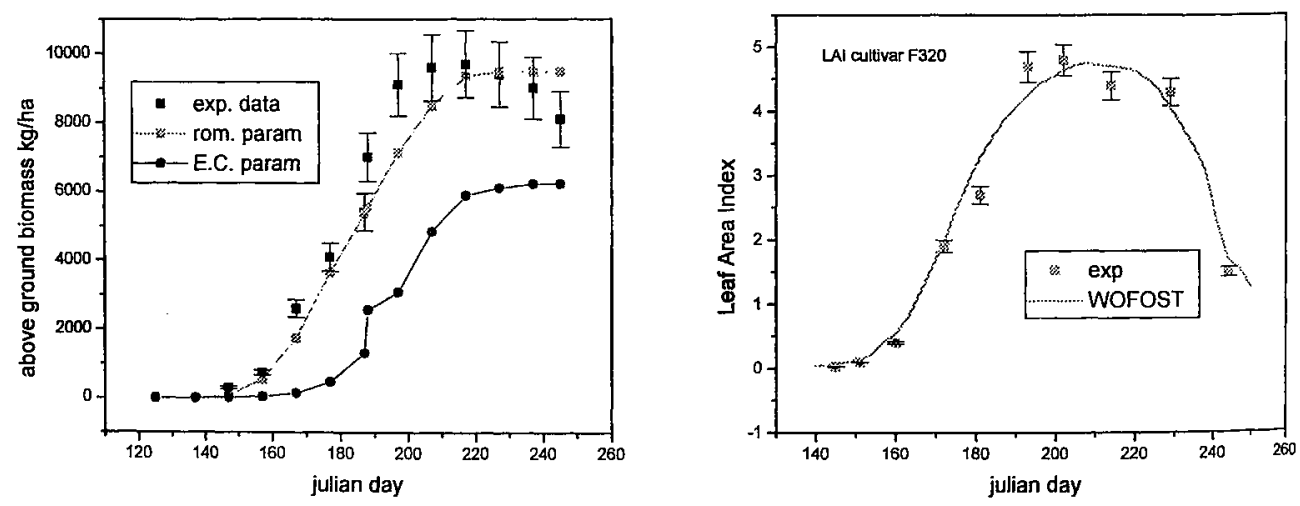

Figure 3 (left) Sunflower above ground biomass, experimental data (exp), WOFOST result for default cultivar (EC param.) and parameters adapted to Romanian cultivars (rom param)

Figure 4 (right) Leaf area index for maize cultivar F320. Experimental data (exp), and WOFOST results with parameters adapted to Romanian cultivar .

The customisation of model parameters was easy to make, because we used existing agricultural research experimental data. The data must contains at least 3-5 points in the reproductive stage of plant growth, in order to have reliable partitioning of dry matter (and hence OBT) to edible plant parts. 
In its present form, the food chain model in RODOS does not consider the actual plant status (e.g. LAI and development stage) but uses mean values estimated over a number of years. This is a weakness which could be avoided in the future by using remote sensing or by running the WOFOST model with daily meteorological data (from plant seedling until day of tritium accidental emission). If the plant parameters in WOFOST are established using data for a specific year, we can assess the mean LAI dynamics by nuning WOFOST for 20 -year local meteorological data (available in each country from meteorological networks). An example of such a multi-year run for irrigated potato is given in Figure 5. From these results, mean LAI dynamics can be extracted as in Table 1.

\section{Potential Crop Production}

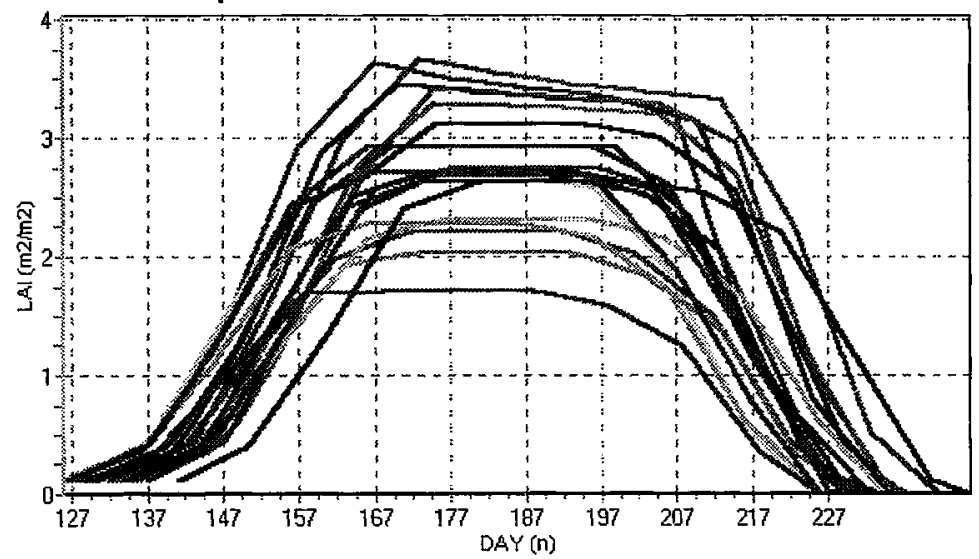

$-W[C, 1970[1]$

$-W C C .1971[1]$

WCC.1972[1]

- WCC.1973(1)

$-W[C .1974(1)$

- WCC. 1975[1]

$-W[C .1976[1]$

- WCC.1977(1)

$-W C C .1978(1)$

$-W C C .1979(i)$

$-W C C .1980(1)$

- w/CC.1981[1]

W... WCC. 1982[1]

$-W C C, 1983(1$

- WCC.1984(1)

- WCC.1985(1)

- WCC. 1986 (1)

$-W C C .1987(1)$

- WCC.1988[1]

- WCC.1989[1]

Figure 5 Consecutive 20 year model results for potato LAI in South Romania Table I Mean dynamics of potato LAI for South Romania

\begin{tabular}{|r|l|l|l|l|l|}
\hline Day of year & 135 & 145 & 167 & 207 & 233 \\
\hline LAI & 0 & 0.5 & 3 & 3 & 0 \\
\hline
\end{tabular}

\subsection{Model parameters for plants not in the WOFOST database}

In order to enhance our ability to model tritium, we have expanded the WOFOST database to include leafy vegetables and tomato, using literature data and local information. Wine grapes are an important economic produce in the area adjacent to the Romanian CANDU reactor. Vines have two peculiarities, they are perennial plants partially covering the ground, a case which is outside the basic approach of. WOFOST. We are unaware of existing crop growth models for grapes and consequently we have investigated the adaptation of the WOFOST model.

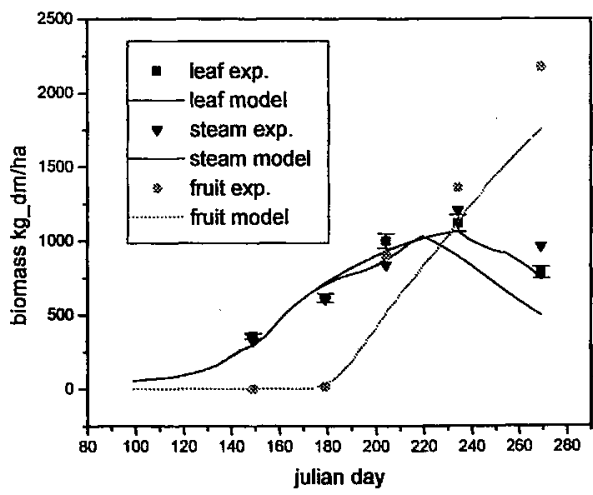

Figure 6 Model predictions compared to experimental data for wine grapes 
An extensive experimental database was obtained for the cultivar "Feteasca Regala" and a wine grape model was built starting with initial model parameters established by a literature review and available experimental data. As for grass and winter wheat in WOFOST, a 'deposit' of active dry matter was considered in early spring in accordance with experimental data. Specific leaf area and mean photosynthetic rate were imposed using experimental measurements. After some iteration, the model parameters have been established giving good reproduction of leaf area index and biomass dynamics, as show in Figure 6. Where, the parameter estimates were compared with literature values and found to be in good agreement. The only major difference is for the maximum photosynthetic rate, which was $40 \%$ lower than reported values. This was imposed by the need to reproduce light absorption in a sparse canopy, using a model for complete cover.

\section{CONCLUSIONS}

Due to the large environmental and physiological variability of tritium transport into the soil-vegetationatmosphere continuum, a predictive model for tritium must avoid calibration using specific tritium experimental data. Its reliability and applicability must reside in the dependence of ${ }^{3} \mathrm{H}$ behaviour on hydrogen and an understanding of processes controlling the hydrogen cycle. Photosynthesis and partitioning of newly formed dry matter are basic processes driving tritium transfer. We have demonstrated that the WOFOST crop growth model: (i) gives predictions comparable with more complex species specific photosynthesis models; (ii) can describe $\mathrm{C} 3$ and $\mathrm{C} 4$ plant growth under various climatic conditions. A procedure to simulate limited fertilisation has been tested and full customisation to Romanian conditions undertaken. The application of the WOFOST crop model to derive tritium transfer from atmosphere to plants will be reported in a forthcoming paper.

\section{Acknowledgements}

Work supported in part by the Centre of Excellence IDRANAP under contract with European Commission ICA1-CT-2000-70023, and by a grant with Royal Society (UK). We are grateful to Nick Beresford (Centre foe Ecology \& Hydrology-Merlewood, UK) and Neil Crout (Nottingham University, UK) for their support. The WOFOST crop model was kindly supplied by Dr. H.L. Boogaard from the DLO Winand Staring Center Institute (Wageningen, Netherlands).

\section{References}

[1] Galeriu D., A. Melintescu , C. Turcanu , W. Raskob, FDMH - The tritium module in RODOS, International Workshop on Environmental Behavior and Biological Effects of Tritium, 8-9 May 2000, Kurri, Kumatori, Osaka, Japan. Ed. M Saito, S. Kimura, and T. Takahashi ISSN 0287-0852 [ KURRIKR-61], p 86.

[2] Jacobs C. M. J., Direct impact of atmospheric $\mathrm{CO}_{2}$ enrichment on regional transpiration, $\mathrm{PhD}$. Thesis, Agricultural University, Wageningen (1994).

[3] Calvet J. C., Agricultural and Forest Meteorology 103, 229 (2000). , Vol. 103, pp.229-247

[4] Boogaard H. L, User's guide for the WOFOST 7.1 crop growth simulation model and WOFOST Control Center 1.5 Technical Document 52 DLO Vinand Staring Centre, Wageningen, 1998

[5] Mynemi R. B. B.D. Ganapol, Remote Sens. Envron. 42, 217 (1992).

[6] Marshall B. P.V. Biscoe, J Exp. Botany 31, 41-48 (1980).

[7] Kimm J. B. Verma, Agricultural and Forest Meteorology 57, 187 (1991).

[8] Collatz G. J. Aust. J. Plant Physiol. 19, 519 (1992).

[9] Dewar R. C., Annals of Botany 78, 125 (1996). 